\title{
Optimizing the Reconstruction of Cardiac Potentials Using a Novel High Resolution Pericardiac Cage
}

\author{
Jake A Bergquist ${ }^{1,2,3}$, Wilson W Good ${ }^{1,2,3}$, Brian Zenger ${ }^{1,2,3,4}$, Jess D Tate ${ }^{1}$, and Rob S MacLeod ${ }^{1,2,3}$ \\ ${ }^{1}$ Scientific Computing and Imaging Institute, University of Utah, SLC, UT, USA \\ ${ }^{2}$ Nora Eccles Cardiovascular Research and Training Institute, University of Utah, SLC, UT, USA \\ ${ }^{3}$ Department of Biomedical Engineering, University of Utah, SLC, UT, USA \\ ${ }^{4}$ School of Medicine, University of Utah, SLC, UT, USA
}

\begin{abstract}
Introduction: Experimental preparations in which cardiac are made typically do not have uniform sampling around the heart surface. Signals captured from the sampled region are extended to the unsampled region before being utilized in computational models. The resulting errors have never been evaluated systematically. We explored this relationship using a novel experimental preparation, and compared the resulting measurements against a set of reconstruction methods.

Methods: Measurements came from a modified Langendorff preparation in which we placed a heart shaped pericardiac cage electrode array around an isolated canine heart within an electrolyte filled torso-tank. We optimized a reconstruction from one region of the cage to another and compared the reconstruction to surface Laplacian interpolation.

Results: Qualitative and calculated error metrics showed lower error between optimized and measured signals, whereas Laplacian interpolation resulted in poorer reconstructions in most cases.

Discussion: We utilized novel recordings to investigate reconstruction of unsampled signals, demonstrating that a sampled subset of signals is sufficient to reconstruct the unsampled subset and that Laplacian interpolation does not achieve the level of accuracy that is possible.
\end{abstract}

\section{Introduction}

Previous studies have shown that an incomplete sampling of the epicardial potentials during experimental preparations creates significant error during computational modeling of the heart.[1] Many experimental preparations use epicardial sock arrays such as the Utah High Density Epicardial Sock (UHDES), which only provides coverage of the ventricles.[2-5] In such cases, the missing (but required) potentials over the atria are approximated using the measured ventricular potentials. A common approach to approximate the missing signals is to interpolate from the measured data often using a surface Laplacian approach, however, it is unclear if interpolation approaches are able to reconstruct the missing atrial signals in a scenario in which only ventricular signals are measured.[6]

To examine this previously unsolved question, we designed a novel torso-tank experimental preparation in which we placed a Utah Pericardiac Cage (UPC) around a Langendorff prepared heart in an instrumented, electrolytic tank shaped like a human torso. The UPC provides 256 recording electrodes that form a closed surface around the entire isolated heart.

Our goal was to leverage the complete coverage of the UPC to determine if missing signals could be reconstructed from measured potentials and if our current reconstruction technique is adequate. We sectioned the UPC into known and unknown potentials analogous to the common experimental scenario in which the superior, atrial region of the cage represented the area with missing potential measurements and the inferior, ventricular region contained the measured potentials. We devised a set of optimization problems that used the measured data to reconstruct the missing data. Preliminary results show that the optimization based approach reconstructed the missing signals with high fidelity, suggesting that these measured signals contain enough information to reconstruct missing atrial potentials. We then tested a spatial Laplacian interpolation approach, which resulted in a worse reconstruction. This study suggests there is enough information contained within routine experimental recordings to reconstruct missing electrical potentials and that a spatial Laplacian interpolation is not adequate at performing this reconstruction in some cases.

\section{Methods}

Torso Tank Preparation: The isolated heart was prepared as described previously.[7,8] Briefly, an isolated ca- 
nine heart was suspended in a Utah Torso Tank (UTT) filled with electrolyte. The isolated heart was perfused by a second canine to provide circulatory support. A Utah High Density Epicardial Sock (UHDES) array was placed over the ventricles of the heart. A Utah Pericardiac Cage (UPC) electrode array consisting of 256 recording electrodes was then placed around the heart (Figure 1). The isolated heart and recording arrays were then lowered into the torso tank. Continuous electrical recordings were captured from the UPC, UHDES, and UTT using a custom multiplexing unit that recorded all electrodes simultaneously at $1 \mathrm{kHz} .[2,3]$

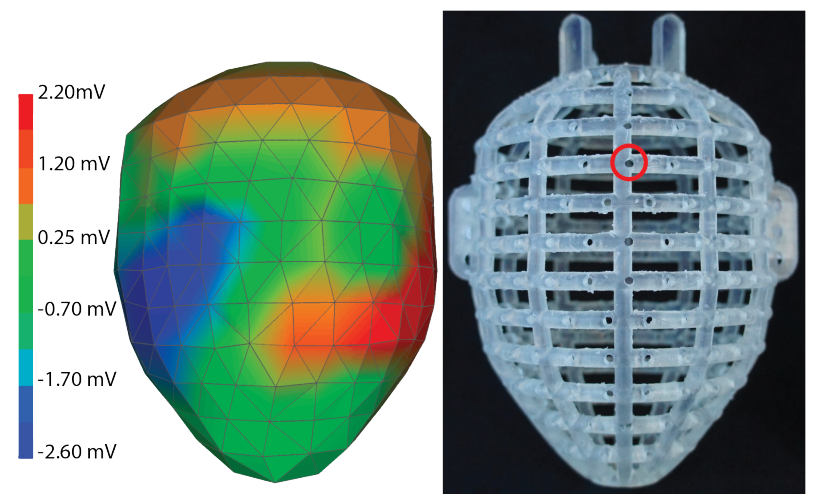

Figure 1. The Utah Pericardiac Cage (UPC). The UPC is a $3 \mathrm{D}$ printed structure that has mounting holes for 256 recording electrodes; one such electrode location is circled in red. The electrode locations connect to form triangles, all of which have approximately equal solid angles with respect to the centroid of the cage. The two halves of the UPC are closed around the isolated heart and both the UPC and heart are immersed in a torso tank filled with electrolyte. The recorded signals can be mapped onto a digitized geometry of the electrode locations. Colors here represent the potential distribution at a time instant 50\% into the QRS of a sinus beat.

Data Processing: Post experiment, the location of the UPC electrodes as well as a subset of UTT and UHDES electrodes were digitized using a Microscribe mechanical digitizer (MicroScribe). The UTT and UPC were registered rigidly, and the UHDES was registered as described previously.[9] The recorded electrograms were processed using "Preprocessing Framework for Electrograms Intermittently Fiducialized from Experimental Recordings" (PFEIFER), an open source MATLAB platform designed to process bioelectrical recordings.[10] We selected the QRST segment of 40 beats from each of the following morphologies to analyze: ventricular paced beats, sinus beats, and beats paced from the right atrium. Pacing artifacts were identified and removed manually.

Reconstruction Formulation: The UPC was divided into a 70 node superior region (comprised of electrodes from the atrial portion of the cage) and a 186 node inferior region (comprised of nodes from the ventricular portion of the cage). The ventricular region $\left(C_{V}\right)$ was treated as the measurements to reconstruct the missing atrial region $\left(C_{A}\right)$. We formulated two optimization approaches for reconstructing the atrial region. Equation 1 shows Optimization formulation 1 (Opt. 1), which minimizes the $L_{2}$ difference of the reconstructed atrial region potentials $\left(D C_{V}\right)$ and the measured atrial cage potentials $\left(C_{A}\right)$ by optimizing the matrix $D$ that maps the ventricular potentials, $C_{V}$, onto $C_{A}$. Equation 2 shows formulation 2 (Opt. 2) which adds an optimization of weights $(F)$ of the derivative of the measured ventricular signals with respect to time $\left(C_{V}^{\prime}\right)$. One beat per morphology (the training data) was used to optimize for the mapping matrices (either $D$, or $E$ and $F$ ). We reconstructed atrial region potentials on the remaining 39 beats per morphology (the testing data) by evaluating the expressions $D C_{V}$ or $E C_{V}+F C_{V}^{\prime}$ respectively for each beat using the mapping matrices optimized for each specific morphology. As a comparison we calculated a surface Laplacian interpolation matrix to reconstruct the atrial potentials using the SCIRun problem solving environment (http://www.sci.utah.edu/cibcsoftware/scirun.html) and applied this Laplacian mapping to each of our testing data beats.

$$
\begin{gathered}
\underset{D}{\operatorname{argmin}}\left\|D C_{V}-C_{A}\right\|^{2} \\
\underset{E, F}{\operatorname{argmin}}\left\|E C_{V}+F C_{V}^{\prime}-C_{A}\right\|^{2}
\end{gathered}
$$

Analysis and Visualization Mean Spatial Correlation over all time points(Pearson's correlation coefficient, SC, Equation 3), Mean Temporal Correlation over all electrodes (TC, Equation 4), and RMS error (RMSE, Equation 5) were used to quantify agreement where $R$ is the reconstructed signal, $M$ is the measured signal, $N$ is the number of recording electrodes, $T$ is the number of time points, and $S D_{M(t)}$ and $S D_{R(T)}$ are the standard deviations of measured and reconstructed signals at a given time point $t$ respectively. For each reconstruction method and testing data beat, $R$ was calculated by evaluating either $D C_{V}, E C_{V}+F C_{V}^{\prime}$, or $L C_{V}$ (where $L$ represents the Laplacian interpolation matrix), for Opt. 1, Opt. 2, or Laplacian interpolation respectively. The optimized mapping matrices used were specific to the training data for each beat morphology. We performed a planar projection of the atrial region of the cage mesh in order to allow for $2 \mathrm{D}$ visualization of the reconstructed signals. The reconstructed signals were mapped onto the nodes of the mesh and visualized in the SCIRun problem solving environment. We chose a time point $50 \%$ into the QRS complex of each beat for visualization to highlight the differences in signal distribution between the reconstruction methods as compared to the measured signals. 


$$
\begin{gathered}
\mathrm{SC}=\frac{\sum_{t=0}^{T} \frac{\left(\left(R(t)-\mu_{R(t)}\right)\left(M(t)-\mu_{M(t)}\right)\right)}{S D_{R(t)} S D_{M(t)}}}{\mathrm{TC}=\frac{\sum_{n=0}^{N} M(n) \circledast \overline{R(n)}}{N}} \\
\mathrm{RMSE}=\sqrt{\frac{\sum\left((R-M)^{2}\right)}{N * T}}
\end{gathered}
$$

\section{Results}

The reconstruction error depended on the beat selected and the reconstruction method. The results summarized in Table 1 show that the two optimized reconstruction methods performed similarly across all three morphologies. In each case the optimized methods provided superior reconstructions compared to the spatial Laplacian interpolation as seen by reduced RMSE, and increased SC and TC. Interpolation resulted in a larger error for the sinus and atrial paced beats than for the ventricular paced beat. Across all reconstruction methods the atrial paced beat resulted in the highest error.

Table 1. Numerical results from the three reconstruction scenarios using the 39 testing data beats per morphology. Average RMSE, Spatial Correlation (SC), and Temporal Correlation (TC) across each morphology are shown.

\begin{tabular}{|lr|r|r|r|}
\hline & & $\begin{array}{r}\text { Ventricular } \\
\text { Paced }\end{array}$ & Sinus & $\begin{array}{r}\text { Atrial } \\
\text { Paced }\end{array}$ \\
\hline & RMSE & $0.10 \mathrm{mV}$ & $0.05 \mathrm{mV}$ & $0.09 \mathrm{mV}$ \\
Opt. 1 & SC & 0.90 & 0.90 & 0.84 \\
& TC & 0.96 & 0.98 & 0.97 \\
\hline & RMSE & $0.10 \mathrm{mV}$ & $0.06 \mathrm{mV}$ & $0.09 \mathrm{mV}$ \\
Opt. 2 & SC & 0.90 & 0.90 & 0.86 \\
& TC & 0.96 & 0.98 & 0.97 \\
\hline & RMSE & $0.31 \mathrm{mV}$ & $0.25 \mathrm{mV}$ & $0.32 \mathrm{mV}$ \\
Lap. & SC. & 0.78 & 0.70 & 0.55 \\
& TC & 0.83 & 0.69 & 0.55 \\
\hline
\end{tabular}

The potential distributions of the Laplacian interpolated and the optimized reconstructed signals varied greatly. The two optimized reconstructions resulted in similar distributions that agreed visually with the measured distributions. The Laplacian interpolated signals showed reasonable agreement with the measured signals for the ventricular paced beat morphology. However, there was striking disagreement between the Laplacian interpolated and measured signals for both the sinus and atrial paced morphologies. The potential distributions for each morphology for one beat from the testing data at 50\% into the QRS complex are displayed in Figure 2.

\section{Discussion}

In this study, we investigated the ability to recapture a subset of missing cardiac potentials from a subset of mea-

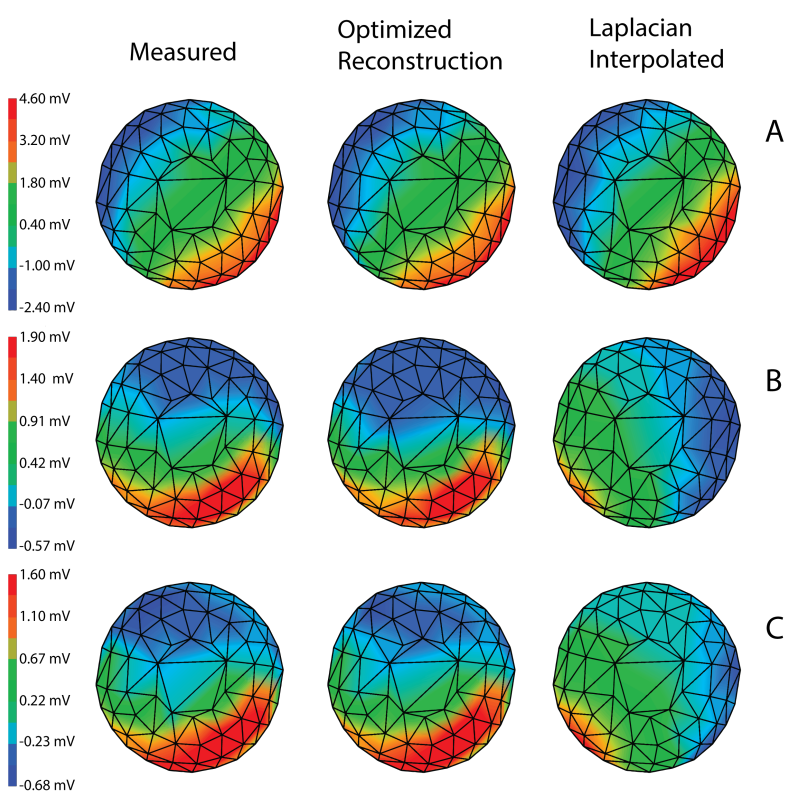

Figure 2. Potentials on the basal subset of the cage for each reconstruction method across three testing data beats. The time point displayed is $50 \%$ into the QRS complex. The measured column shows the signals measured from the cage. The optimized reconstruction column shows potentials from the Opt. 1 method. The Laplacian Interpolation column shows the potentials from the spatial Laplacian interpolation. Rows A, B, and C, show a ventricular paced, sinus, and atrial paced beat respectively.

surements by use of either spatial Laplacian interpolation or data driven optimization. We utilized a novel recording array, the Utah Pericardial Cage (UPC), to record from a closed surface around the entire heart during a torso-tank experiment. By artificially dividing these measured potentials into an ventricular subset and an atrial subset, we assessed the reconstruction scenario common during experimental preparations in which measurements come from the accessible ventricular epicardium. We found that using an optimization based approach that incorporated the measurements in both subsets of the cage, we could reconstruct the atrial subset from the ventricular subset with high fidelity. We compared these reconstructions to a spatial Laplacian interpolation from the measured sources and found that optimized performed better across all beat morphologies tested.

We found that the atrial paced beat resulted in the highest errors across all reconstruction methods. This may be attributed to the complexity of an atrially paced beat. As expected, the ventricular paced beat resulted in the lowest errors, with even the spatial Laplacian interpolation providing comparable results to the optimized methods. This suggests that for simple beat morphologies such as a ven- 
tricular paced beats, spatial Laplacian interpolation could be sufficient.

This study leveraged an ideal scenario using the optimization problem in which the desired missing data was available for at least one beat and could be incorporated into the formulation for future reconstructions, which is often not the case in typical experimental preparations. This study showed that missing signals during experiments can be reconstructed from measured signals with high fidelity if reconstructed properly. Thus, the results from the optimization approaches provide a benchmark of "ideal reconstruction" against which to compare other methods such as spatial Laplacian interpolation. This was the first study to explicitly test the robustness of any interpolation method by leveraging the novel UPC recording array. Such optimization approaches could also be reformulated to incorporate inverse computed potentials or other relevant measurements in place of the measured missing data. The optimization could also be reformulated to minimize the difference between the fully reconstructed and measured potentials forward computed to another measurement surface (such as a torso surface) and the measured potentials at that other surface, as a way to optimize the reconstruction matrix. Thus, this study lays out a general framework for the investigation and potential optimization of reconstruction of unsampled cardiac sources from a set of measured sources on a variety of measurement surfaces. Future plans include the investigation of the trends seen in the optimized mapping matrices to formulate a general strategy using sampled ventricular signals to reconstruct unsampled atrial signals. The optimization framework we developed in this study will be applied to reconstruct of unsampled epicardial sources from experiments that utilize partial epicardial recording arrays, such as the Utah High Density Epicardial Sock (UHDES).

Our study does have limitations. While the UPC forms a closed recording surface around the heart, the resolution of electrical recordings may not sample its electrical sources adequately in certain circumstances. Consequently, complex and subtle aspects of the cardiac source may be missed by these recordings. Additionally, an optimized mapping matrix found using one beat morphology did not apply generally to different beat morphologies. This finding suggests that the optimization formulation could be improved to account for variability in beat morphology.

\section{Acknowledgments}

Support for this research came from the NIHNIGMS Center for Integrative Biomedical Computing (www.sci.utah.edu/cibc), NIH NIGMS grant no.P41GM103545 and the Nora Eccles Treadwell Foundation for Cardiovascular Research.

\section{References}

[1] Tate J, Gillette K, Burton B, Good W, Zenger B, Coll-Font J, Brooks D, MacLeod. R. Reducing error in ecg forward simulations with improved source sampling. Front Physiol 2018;9(1304):1-16.

[2] Burton B, Aras K, Good W, Tate J, Zenger B, MacLeod. R. A framework for image-based modeling of acute myocardial ischemia using intramurally recorded extracellular potential. Annal Biomed Eng 2018;46(9):1325-1336.

[3] Zenger B, Good W, MacLeod R, Tate J, Sharma V, Bergquist J. Electrocardiographic comparison of dobutamine and bruce cardiac stress testing with high resolution mapping in experimental models. 2018 Computing in Cardiology Conference CinC 2019;45:2-5.

[4] Good W, Erem B, Zenger B, Coll-Font J, Brooks H, MacLeod. R. Temporal performance of laplacian eigenmaps and $3 \mathrm{~d}$ conduction velocity in detecting ischemic stress. J Electrocardiol 2018;51(6S):116-120.

[5] Bear L, Cheng L, LeGrice I, Sands G, Lever N, Paterson D, Smaill B. Forward problem of electrocardiography: is it solved? Circ Arrhythm Electrophysiol Jun 2015;8(3):677684.

[6] Huiskamp G. Difference formulas for the surface Laplacian on a triangulated surface. Journal of Computational Physics 1991;95(2):477-496. ISSN 10902716.

[7] MacLeod R, Taccardi B, Lux R. Electrocardiographic mapping in a realistic torso tank preparation. In Proceedings of the IEEE Engineering in Medicine and Biology Society 17th Annual International Conference. IEEE Press, 1995; 245-246.

[8] MacLeod R, Ni Q, Punske B, Ershler P, Yilmaz B, Taccardi. B. Effects of heart position on the body-surface ECG. J Electrocardiol Feb. 2000;33((supp)):229-238.

[9] Bergquist JA, Good WW, Zenger B, Tate JD, MacLeod RS. GRÖMeR: A Pipeline for Geodesic Refinement of Mesh Registration. In Coudière Y, Ozenne V, Vigmond E, Zemzemi N (eds.), Functional Imaging and Modeling of the Heart, volume 11504 LNCS. Cham: Springer International Publishing. ISBN 978-3-030-21949-9. ISSN 16113349 , 2019; 37-45.

[10] Rodenhauser A, Good W, Zenger B, Tate J, Aras K, Burton B, MacLeod R. Pfeifer: Preprocessing framework for electrograms intermittently fiducialized from experimental recordings. J Open Source Software 2018;3(21):472.

Address for correspondence:

Jake A. Bergquist

72 Central Campus Dr, Salt Lake City, UT, USA 84112

jbergquist@sci.utah.edu 